\title{
Order parameter phase locking as a cause of a zero bias peak in the differential tunneling conductance of bilayers with electron-hole pairing
}

\author{
A. I. Bezugly * \\ NSC Kharkov Institute of Physics and Technology, 61108, Kharkov, Ukraine \\ S. I. Shevchenkd \\ B. I. Verkin Institute for Low Temperature Physics and Engineering \\ National Academy of Sciences of Ukraine, Kharkov 61103, Ukraine
}

\begin{abstract}
In $n-p$ bilayer systems an exotic phase-coherent state emerges due to Coulomb pairing of $n$-layer electrons with $p$-layer holes. Unlike Josephson junctions, the order parameter phase may be locked by matrix elements of interlayer tunneling in $n-p$ bilayers. Here we show how the phase locking phenomenon specifies the response of the electron-hole condensate to interlayer voltages. In the absence of an applied magnetic field, the phase is steady-state (locked) at low interlayer voltages, $V<V_{c}$, however the phase increases monotonically with time (is unlocked) at $V>V_{c}$. The change in the system dynamics at $V=V_{c}$ gives rise to a peak in the differential tunneling conductance. The peak width $V_{c}$ is proportional to the absolute value of the tunneling matrix element $\left|T_{12}\right|$, but its height does not depend on $\left|T_{12}\right|$; thus the peak is sharp for small $\left|T_{12}\right|$. A sufficiently strong in-plane magnetic field reduces considerably the peak height. The present results are in qualitative agreement with the zero bias peak behavior that has recently been observed in bilayer quantum Hall ferromagnets with spontaneous interlayer phase coherence.
\end{abstract}

The idea that in bilayer $n-p$ structures consisting of an electron- conductivity layer ( $n$-layer) and a hole-conductivity layer ( $p$-layer) the Coulomb attraction of electrons and holes may lead a formation of electron-hole pairs with spatially separated components was put forward rather long ago $[1,2]$. As a result of Bose-Einstein condensation of these pairs, there arises a peculiar superfluid (phase-coherent) state, in which a dissipationless motion of pairs gives rise to equalin-magnitude and oppositely directed supercurrents in $n$ - and $p$-layers. At present, two variants of the systems have been realized experimentally, where an excitonic condensate with spatially separated components is formed. In both cases, these are closely lying GaAs/AlGaAs double quantum wells, where either interwell excitons are excited by a laser pulse [3, 4], or two-dimensional electron layers are formed due to doping. In the last case, the electron layers must be placed in a strong magnetic field, normal to the layers, such that the total filling factor should be $\nu_{T}=\nu_{1}+\nu_{2}=1$ [5]. Since all these systems have one and the same exciton mechanism for the interlayer phase coherence [6], the physical properties of these systems in the coherent state must be qualitatively similar.

The present paper has mainly been stimulated by recent impressing experiments of Spielman et al. [7, 8] who have found that if a bilayer electron system transits into a phase-coherent state (in which the quantum Hall effect is observed at $\nu_{T}=1$ ), then this transition is accompanied by a sharp rise in the differential tunneling conductance $G_{T}$ at low interlayer voltages $V$. As the temperature is lowered, this peak of tunneling conductance remains of a finite height and width in contrast to the tunneling conductance peak of the Josephson junction. In the parallel to the layers magnetic field $H$ the peak height decreases the more drastically, the higher is the field, and at $H>0.6 \mathrm{~T}$ the peak becomes practically indistinguishable.

A number of papers have been devoted to theoretical interpretation of the experimental results obtained by Spielman et al. For example, Fogler and Wilczek [9] have treated the tunneling conductance peak as a consequence of the Josephson effect in a long inhomogeneous junction. In refs. $[10,11]$, the interpretation of the peak has been based on the notion of the finite time of phase coherence. Joglekar and MacDonald [12] have performed both phenomenological and microscopic calculations of tunneling conductance $G_{T}$ value at $V=0$. In ref. [13], $G_{T}(V, H)$ was calculated using the phenomenological equation similar to the Landau-Lifshitz equation for the magnetic moment. Such a diversity of theoretical approaches in the interpretation of experiment $[7,8]$ gives impetus to a consistent microscopic consideration of the dynamics of phase-coherent bilayer systems, this being just the subject of the present paper. Though we consider the $n-p$ system in the absence of a perpendicular magnetic field, the exciton nature of the collective state in all the above-mentioned systems encourages us to believe that the present results provide a qualitative description of experiments by Spielman et al. [7, 8].

An important but still not completely resolved problem for the systems with electron-hole pairing is the problem of phase locking by interband transitions [14], which coincide with interlayer tunneling transitions in the systems under consideration. The tunneling transitions lift the degeneracy in the phase of the order parameter, thereby locking the phase and making it equal to the phase of tunneling matrix elements. The last statement is valid in the absence of a parallel to the layers magnetic field. Kulik and one of the present authors [15] have shown that in the magnetic field parallel to the layers the phase is locked only in the fields $H<H_{c 1}$. (The critical field $H_{c 1} \propto\left|T_{12}\right|^{1 / 2}$, where $T_{12}\left(=\left|T_{12}\right| e^{i \chi}\right)$ is the matrix element of interlayer tunneling). At $H>H_{c 1}$ the phase locking is lifted and the phase 
monotonically changes in the direction normal to the field, this giving rise to spatial oscillations of tunneling current (vortex state). The phase locking phenomenon appears to exert an essential effect not only on the thermodynamic properties of $n-p$ systems, but also on their kinetics.

Relying on the microscopic approach, the present paper deals with the response of phase-coherent $n-p$ system to the interlayer voltage $V$. We demonstrate that similarly to the existence of the critical field $H_{c 1}$, in the case under consideration there exists the threshold voltage $V_{c}\left(\propto\left|T_{12}\right|\right)$ that quantitatively characterizes the degree of phase locking in the $n-p$ system. At low voltages, $V<V_{c}$, the order parameter phase is locked (steady-state), and the direct tunneling current is proportional to $V$. The Ohmic character of a spatially uniform tunneling current at $V<V_{c}$ means that in the phase-coherent $n-p$ system there is no dc Josephson effect [16]. (The absence of the dc Josephson effect in the two- layer electron system has been established by Joglekar and MacDonald [12]). At voltages $V>V_{c}$, the phase monotonically changes with time, and this results in the tunneling current oscillations with frequency $\omega=e \sqrt{V^{2}-V_{c}^{2}}$ (here $e$ is the elementary charge, $\hbar=1$ ) So, at $V>V_{c}$ the $n-p$ system retains the essential feature of the ac Josephson effect in superconductors, namely, the tunneling current oscillations at a constant applied voltage. At the same time, the dissipative character of the oscillating tunneling current (see below), the nonuniversality of the voltage dependence of $\omega$, and the presence of threshold voltage $V_{c}$ are specific to phase-coherent bilayer $n-p$ systems.

Further on, we show that the above-described "liberation" of the order parameter phase at $V=V_{c}$ results in a sharp peak of $G_{T}(V)$, the height of which is independent of $\left|T_{12}\right|$, and its width is equal to $2 V_{c}$, i.e., for small $\left|T_{12}\right|$ the peak will be high and sharp. Thus, in our opinion, the nature of the tunneling conductance peak observed in the experiments of Spielman et al. is closely connected with the phenomenon of order parameter phase locking by tunneling transitions. The experimentally observed suppression of $G_{T}(V)$ peak with an increasing parallel magnetic field [8] also lends support in favor of this interpretation, because, as indicated above, a sufficiently strong in-plane magnetic field eliminates the phase locking.

We are now coming to the analysis of the dynamics of a phase-coherent $n-p$ system in the limit of a high pair density, when the average distance between the electron-hole pairs is small as compared to the characteristic pair size. The advantage of the high-density limit lies in the possibility of considering the phase-coherent system dynamics in the gapless state, when the gap in the excitation spectrum becomes zero under the action of strong depairing, and the order parameter $\Delta$ is reduced but remains non-zero [17]. For the $n-p$ bilayer the order parameter is proportional to the average $\left\langle\psi_{1}(\mathbf{r}, t) \psi_{2}^{+}(\mathbf{r}, t)\right\rangle$, where $\psi_{i}^{+}(\mathbf{r}, t)$ is the electron creation operator in the layer $i$. An essential simplification consists in the fact that the absence of the gap makes it possible to describe the dynamics of the phase-coherent system only in terms of the complex order parameter $\left(\Delta=|\Delta| e^{i \theta}\right)$ without involving the dynamics of the quasiparticle distribution function.

The dynamic equation for the order parameter of the $n-p$ system was derived by the Green function technique in our paper [18] and has the following form:

$$
-(\dot{\Delta}-i e V \Delta)+\left\{A-B|\Delta|^{2}+D\left[\frac{\partial}{\partial \mathbf{r}}+\frac{i e}{c}\left(\mathbf{A}_{1}-\mathbf{A}_{2}\right)\right]^{2}\right\} \Delta+\frac{T_{12}}{\zeta \tau}=0 .
$$

The equation obtained is in a perfect agreement with the general theory of a relaxation of an order parameter near a point of a phase transition of the second kind (see, for instance [19]). In accordance with this theory a state of a physical system under a phase transition of the second kind can be described by an order parameter, that is nonzero below the transition point and equal to zero above this point. An equilibrium value of the order parameter can be found from the condition that a variation of a corresponding thermodynamic potential is equal to zero. In the absence of the interband hybridization the thermodynamic potential for the condensate of electron-hole pairs with spatially separated components can be presented in the form

$$
F=\int\left\{D\left|\left[-i \frac{\partial}{\partial \mathbf{r}}-\frac{e}{c}\left(\mathbf{A}_{1}-\mathbf{A}_{2}\right)\right] \Delta\right|^{2}+A|\Delta|^{2}+\frac{1}{2} B|\Delta|^{4}\right\} d \mathbf{r}
$$

Expression (2) is similar to the thermodynamic potential for Cooper pairs in the Ginzburg-Landau theory, but here the term $2 e \mathbf{A}$ is replaced by the term $e\left(\mathbf{A}_{1}-\mathbf{A}_{2}\right)$. Such a modification is quite natural. Indeed, for the case of electronhole pairs with spatially separated components an electron in the layer 1 "sees" the vector potential $\mathbf{A}_{1}$, while a hole in the layer 2 "sees" the vector potential $\mathbf{A}_{2}$. Since the signs of the electron and hole charges are different the vector potentials $\mathbf{A}_{1}$ and $\mathbf{A}_{2}$ are subtracted from each other in eq. (2). In the equilibrium the order parameter $\Delta(\mathbf{r})$ is found from the condition $\delta F / \delta \Delta(\mathbf{r})=0$. At small deviation from the equilibrium, when the derivative $\delta F / \delta \Delta(\mathbf{r})$ is nonzero but a small one, the order parameter relaxation rate (the derivative $\partial \Delta / \partial t$ ) is also small. In the mean field approximation these two derivatives should be proportional to each other. But it is necessary to take into account that due to the gauge invariance of the theory the derivative $\partial / \partial t$ can enter into the equation in a combination with the term $i e\left(V_{1}-V_{2}\right)$, where $V_{1}$ and $V_{2}$ are the electrostatic potentials in the layer 1 and 2, correspondingly. As a 
result in the absence of the interband hybridization one arrives to the equation (11), where $T_{12}=0$. In the presence of the interband hybridization the Hamiltonian of the system contains the terms linear in the order parameter $\Delta$ and in the matrix elements $T_{12}$ and the corresponding conjugate terms (and it means that the thermodynamic potential contains the same terms). These terms play the role of a source of electron-hole pairs. They are analogous to the terms that appear in the Hamiltonian of a ferromagnet in an external magnetic field. For the case of a magnet it results in an appearance of a term linear in the magnetic field in the equation for the order parameter. Since for the system considered the matrix element $T_{12}$ is analogous to the magnetic field, a term linear in $T_{12}$ should appear in the equation for the order parameter in the presence of the interband hybridization. We see that eq. (II) contains this term, indeed. The microscopic analysis shows that in spite of the phenomenological arguments presented look quite general, in reality, eq. (11) is valid only in a rather narrow interval of the impurity concentration in similarity with the Gor'kov-Eliashberg equation for the superconductors with paramagnetic impurities [20].

In the gapless situation under consideration, the coefficients of the dynamic Ginzburg-Landau equation (11) have the forms $A(T)=\left(2 \pi^{2} / 3\right) \tau\left(T_{c}^{2}-T^{2}\right), B=4 m \tau / 3 M, D=p_{0}^{2} \tau / M^{2}$ [18]. Here $\tau$ is the electron elastic scattering time ( for simplicity, it is considered equal to the hole elastic scattering time), $T$ is the temperature $\left(k_{B}=1\right), T_{c}$ is the critical temperature, $M=m_{1}+m_{2}$ is the pair mass, $m=m_{1} m_{2} / M$ is the reduced mass of pair, $p_{0}$ is the Fermi momentum of electrons and holes, $\zeta$ is the dimensionless constant of the Coulomb interaction [2]. It should be noted that eq. (II) is derivated by expanding of the anomalous Green function as a power series in $\left(\Delta / T_{c}\right)$ [18]. Since a term linear in the matrix element $T_{12}$ appears in the expression for the order parameter, it is necessary that $\left|T_{12}\right| \ll T_{c}$ for the validity of eq. (11).

At low fields and currents, the modulus of the order parameter varies only slightly in space and time. Assuming $|\Delta|$ to be constant equal to $\Delta_{0}$, the imaginary part of eq. (1) can be written as follows

$$
\dot{\phi}-D \frac{\partial}{\partial \mathbf{r}}\left(\frac{\partial \phi}{\partial \mathbf{r}}-\frac{2 \pi d}{\Phi_{0}}[\mathbf{H n}]\right)-e V+e V_{c} \sin \phi=0 .
$$

Here, the gradient-invariant phase $\phi=\theta-\chi-\left(2 \pi d / \Phi_{0}\right) A_{z}$ is introduced, $d$ is the interlayer distance, $\Phi_{0}=h c / e$ is the magnetic flux. The unit vector $\mathbf{n}=(0,0,1)$ is normal to the layers and is directed from layer 1 (electron layer) to layer 2 (hole layer). The threshold voltage $V_{c}=\left|T_{12}\right| /\left(e \zeta \tau \Delta_{0}\right)$.

It is readily seen that in the uniform case eq. (3) for the phase $\phi$ is different from the equation $\dot{\phi}=e V$ that appears in a number of papers and is treated as the Josephson relation for phase-coherent bilayer systems. The occurrence of the term proportional to $\left|T_{12}\right|$ in the dynamic equation for the phase radically changes the solutions of this equation. Thus, in the absence of external fields the stable steady-state and uniform solution of eq. (3) is $\phi=0$, i.e., $\theta=\chi$, and this means that the interlayer tunneling transitions hold the order parameter phase locked. Below, we consider in detail how the phase locking phenomenon influences the dynamic properties of $n-p$ systems.

We start from the analysis of dynamics of the $n-p$ system in the phase-coherent state for the spatially uniform case in the absence of the magnetic field. Let the $n-p$ tunneling junction is incorporated into the electrical circuit having the resistance $R$ and the voltage source $\mathcal{E}$. The resulting voltage $V$ across the $n-p$ tunneling junction determines the difference of electrochemical potentials of the layers and thereby dictates the carrier density in $n-$ and $p$-layers. If $\delta n$ is the deviation of the electron density from the equilibrium one, then the equality $\mathrm{eV}=-\delta n / N_{*}(0)$ is valid, where the renormalized density of states on the Fermi surface is $N_{*}(0)=N(0)\left(1+\frac{e^{2} m}{\pi C}\right)^{-1}$ (C is the capacity of the bilayer system related to the unit area; $N(0)=m / \pi)$.

In the approximation linear in $T_{12}$ the density of tunneling current from layer 1 to layer 2 is equal to $J=J_{c} \sin \phi$, where $J_{c}=4 e N(0)\left|T_{12}\right| \Delta_{0} / \zeta[18]$. The charge balance equation can be written as

$$
e S \delta \dot{n}+\frac{\mathcal{E}-V}{R}-I_{c} \sin \phi=0,
$$

where $S$ is the area of the $n-p$ junction, and $I_{c}=S J_{c}$. Though below we assume $\mathcal{E}=$ const, it should be noted that eq. (44) also holds for the time-dependent voltage source.

Making use of the relationship between $\delta n$ and $V$, from eqs. (3) and (4) one can derive the second-order equation for the phase $\phi$. In terms of dimensionless variables, this equation takes on the following form, well known in the theory of Josephson junctions

$$
\ddot{\phi}+\frac{1}{\sqrt{\beta}}(1+\epsilon \cos \phi) \dot{\phi}+\sin \phi=\rho .
$$

Here, the following dimensionless parameters are introduced: $\beta=e \mathcal{E}_{c} t_{0}, \epsilon=e V_{c} t_{0}, \rho=\mathcal{E} / \mathcal{E}_{c}$, where $\mathcal{E}_{c}=V_{c}+I_{c} R$, and $t_{0}=e^{2} N_{*}(0) R S$. The time is measured in the $1 / \omega_{0}$ units, where $\omega_{0}=\left(e \mathcal{E}_{c} / t_{0}\right)^{1 / 2}$. 
Despite the coincidence of eq.(5) with the dynamic equation for the phase difference across the Josephson junction, the different meaning of the parameter $\rho$ entering into these equations leads (as it will be seen from what follows) to a substantially different behavior of $n-p$ systems and Josephson junctions.

A detailed analysis of dynamic states of the system described by eq. (5) was performed by Belykh et al. [21] Not going into details of that analysis, we shall mention its main results. For each value of the parameter $\epsilon$ one can find the corresponding number $\beta_{1}$. At $\beta>\beta_{1}$ (large resistances $R$ ), the range of $\rho$ values is split into three adjacent intervals: $0<\rho<\rho_{c}, \rho_{c}<\rho<1, \rho>1\left(\rho_{c}(\beta, \epsilon)\right.$ is the bifurcation value of the parameter $\rho$ [21]). In the first interval, there is only one stable solution $\phi=\arcsin \rho$; in the third interval the only stable state is the limiting cycle embracing the phase cylinder. In the intermediate (second) interval the both solutions, $\phi=\arcsin \rho$ and the limiting cycle, are stable. This non-uniqueness of the solution of eq. (5) results in the hysteresis of current-voltage characteristic (CVC) at $\beta>\beta_{1}$. For $\beta<\beta_{1}$ (low resistances $R$ ) the stable solutions will be $\phi=\arcsin \rho$ at $0<\rho<1$ and the limiting cycle at $\rho>1$, while the interval $\rho$ with two stable states drops out. Correspondingly, at $\beta<\beta_{1}$ the CVCs have no hysteresis.

Further on, we find the CVC and the differential tunneling conductance of the $n-p$ system in a simple, but physically rather demonstrative, case $R=0$. In this limit, no distinction may be made between $V$ and $\mathcal{E}, V_{c}$ and $\mathcal{E}_{c}$, and the dynamics of the system may be analyzed relying on eq. (3) (without spatial derivatives). Since in the case considered we have $\beta<\beta_{1}$, then the hysteresis of the CVC is absent.

If the system is spatially uniform and the voltage $V$ does not depend on the time the equation (3) can be integrated. One can see that for $V<V_{c}$ the equation (3) has the time independent solution $\phi_{0}=\arcsin V / V_{c}$. In such a case the tunnel current, that does not depend on time as well, is equal to $I_{c} \sin \phi_{0}=I_{c} V / V_{c} \equiv V / R_{c}$. This current is proportional to the voltage $V$ applied and it is a usual dissipative current.

The corresponding tunneling conductance is given by

$$
G_{T}=\frac{d I}{d V}=R_{c}^{-1}=4 e^{2} N(0) \tau \Delta_{0}^{2} S
$$

Note that at $V<V_{c}$ the tunneling conductance is constant and is independent of the tunneling matrix element $\left|T_{12}\right|$ value. This independence of tunneling conductance from $\left|T_{12}\right|$, and also its proportionality to $\Delta_{0}^{2}(T)$ are in agreement with the result of Joglekar and MacDonald [12] for $G_{T}$ at $V=0$.

In case of $V>V_{c}$ the integration yields the tunnel current equals to

$$
I(t)=2 I_{c} \frac{\tan \frac{\phi(t)}{2}}{1+\tan ^{2} \frac{\phi(t)}{2}}
$$

where

$$
\tan \frac{\phi(t)}{2}=V_{c} / V+\sqrt{1-\left(V_{c} / V\right)^{2}} \tan \left[\frac{e}{2}\left(V^{2}-V_{c}^{2}\right)^{\frac{1}{2}}\left(t-t_{0}\right)\right]
$$

One can see that the interlayer current oscillates with the frequency $\omega=e\left(V^{2}-V_{c}^{2}\right)^{1 / 2}$ and this current is not a sinusoidal one. Due to non-sinusoidal character of the oscillations the average value of the tunnel current is nonzero. The average current is the function on the voltage $V$.

$$
I=\left(I_{c} / V_{c}\right)\left(V-\sqrt{V^{2}-V_{c}^{2}}\right) .
$$

The behavior of the system considered is similar to the behavior of a Josephson junction between two superconductors in a circuit, where the junction is connected in series with a resistor and a voltage generator. But in the case considered the essential difference is that the resistor (with $R_{c}=V_{c} / I_{c}$ ) is embedded in the junction and it cannot be deleted from the circuit. There is not any transverse superconductivity in the systems considered.

Since according to eq. (9) the tunneling current decreases with an increasing voltage, the differential tunneling conductance at $V>V_{c}$ is negative:

$$
G_{T}(V)=-\left(I_{c} / V_{c}\right)\left[V\left(V^{2}-V_{c}^{2}\right)^{-1 / 2}-1\right] .
$$

The conductance $G_{T}(V)$ has its maximum (constant) value at $|V|<V_{c}$ and the discontinuity points at $V= \pm V_{c}$. At $|V|>V_{c}$, as $|V|$ increases, the tunneling conductance monotonically tends to zero, remaining negative. If we take 
into account the fluctuation smoothing of the CVC, then the dependence of $G_{T}$ on $V$ will look like a smooth curve with the maximum at $V=0$ (approximately $2 V_{c}$ in width) and two minima at $V \approx \pm V_{c}$. It is just this behavior of the $G_{T}(V)$ curve that was observed in experiment [8] in the absence of the magnetic field parallel to the layers.

It should be noted that both at $V>V_{c}$ and $V<V_{c}$ the spatially uniform tunneling current is dissipative. The reason for the dissipation lies in the fact that the uniform interlayer current causes the order parameter phase to deviate from its equilibrium value and a continuous input of energy is required to maintain this nonequilibrium state.

Let now the bilayer $n-p$ structure be placed in a magnetic field $\mathbf{H}$ parallel to the layers and directed along the $x$-axis. If $H>H_{c 1}=\left(2 \Phi_{0} / \pi^{2} d\right)\left(J_{c} M / e n_{s}\right)^{1 / 2}$ (the two-dimensional density of the pairs $\left.n_{s}=4 p_{0}^{2} N(0)\left(\tau \Delta_{0}\right)^{2} / M\right)$, then the magnetic field between the layers has a nonuniform (vortex) component. We shall show that the CVCs of the $n-p$ system in the magnetic field strongly differ from the CVCs in the zero field and are substantially different at both low and high resistances $R$. In the limiting case $R=0$ (and $H \gg H_{c 1}$ ), the solution of eq. (3) can be derived using the perturbation theory. Putting $\phi=\phi_{0}+\phi_{1}$, where $\phi_{0}=k y+\omega t\left(k=2 \pi d H / \Phi_{0}, \omega=e V\right)$ and taking into account the correction term $\phi_{1}$ (proportional to a small $T_{12}$ value) as a perturbation, we obtain the following expression for the average tunneling current density:

$$
J=J_{c} \frac{e V_{c}}{2} \frac{\omega}{\left(D k^{2}\right)^{2}+\omega^{2}} .
$$

So, for $R=0$ the CVC has a wide diffusion maximum at $\omega=D k^{2}$.

At high $R$ values, the charge transport from one layer to the other over the electrical circuit is insignificant, and the electron density dynamics in layer 1 is determined by the continuity equation

$$
e \delta \dot{n}=\operatorname{div}_{2} \mathbf{j}+J_{c} \sin \phi
$$

where $\operatorname{div}_{2} \mathbf{j}$ denotes the two-dimensional divergence of the intralayer current $\mathbf{j}=-\frac{e n_{s}}{M}\left(\frac{\partial \phi}{\partial \mathbf{r}}-\frac{2 \pi d}{\Phi_{0}}[\mathbf{H n}]\right)$. In the assumption that $e V \ll \tau \Delta_{0}^{2}$, the above-described perturbation-theory procedure yields the following equation for $\phi_{1}$ :

$$
\ddot{\phi}_{1}-D \frac{\partial^{2} \dot{\phi}_{1}}{\partial^{2} \mathbf{r}}-u_{0}^{2} \frac{\partial^{2} \phi_{1}}{\partial^{2} \mathbf{r}}=-\frac{J_{c}}{e N_{*}(0)} \sin \phi_{0},
$$

where $u_{0}=\left(n_{s} / M N_{*}(0)\right)^{1 / 2}$. Unlike the $R=0$ case, the left-hand part of eq. (13) has a wave character rather than a diffusion character. Correspondingly, the expression for the average tunneling current density

$$
J=J_{c} \frac{1}{2 \lambda_{J}^{2}} \frac{\omega \alpha k^{2}}{\left(\omega^{2} / u_{0}^{2}-k^{2}\right)^{2}+\left(\omega \alpha k^{2}\right)^{2}}
$$

has the resonance at $\omega=u_{0} k$, the width of which is determined by the attenuation $\alpha=D / u_{0}^{2}$. This resonance results from the coincidence between the plasmon velocity $u_{0}$ in the bilayer structure and the velocity of the magnetic-field vortices. The parameter $\lambda_{J}$ equals $\left(e n_{s} / M J_{c}\right)^{1 / 2}$.

From relations (11) and (14) it follows that at $H \gg H_{c 1}$ the $G_{T}(0)$ value is proportional to a small $\left|T_{12}\right|^{2}$ value, i.e., the differential tunneling conductance peak (occurring at $H=0$ ) is strongly suppressed. The reason for this suppression lies in the fact that at $H>H_{c 1}$ the phase $\phi$ monotonically varies with the coordinate, and in this case eq. (3) has no stationary solution at finite voltage, i.e., no phase locking arises.

Thus, the present work has demonstrated in the frame of the consistent microscopic approach that in phase-coherent bilayer $n-p$ systems the known phenomenon of order parameter phase locking by matrix elements of tunneling $T_{12}$ leads to a sharp peak in differential tunneling conductance $G_{T}(V)$ at $V=0$. The peak height is independent of $\left|T_{12}\right|$ and its width is proportional $\left|T_{12}\right|$, i.e., at weak tunneling the peak is high and sharp. These results are in qualitative agreement with the peculiarities of $G_{T}(V)$ observed in electron bilayer systems in the regime of an integral quantum Hall effect at the total filling factor $\nu_{T}=1$. We stress once again that though the theory developed here describes the $n-p$ system without a transverse magnetic field, the present results are in qualitative agreement with the data from experiments on electron bilayer systems in a strong transverse magnetic field. This agreement does not seem to be accidental. The reason is that most likely the strong magnetic field does not affect the structure of the equation that defines the dynamics of the order parameter, but only changes the values of coefficients entering into this equation.

This work was supported by the INTAS program, grant No 01-2344. 
References

1. Yu.E. Lozovik and V.I. Yudson, Zh. Eksp. Teor. Fiz 71, 738 (1976) [ Sov. Phys. JETP 44, 389 (1976)].

2. S.I. Shevchenko, Fiz. Nizk. Temp.2, 505 (1976) [Sov. J Low Temp. Phys. 2, 251 (1976)].

3. L.V. Butov, C.W. Lai, A.L. Ivanov, A.C. Gossard, and D.S. Chemia, Nature, 417, 47 (2002); L.V. Butov, A.C. Gossard, and D.S. Chemia, Nature, 418, 751 (2002).

4. A.V. Larionov, V.B. Timofeyev, J. Hvam, K. Soerensen, Pis'ma v ZhETF 75, 233 (2002) [JETP Letters 75, 200 (2002)]; A.A. Dremin, V.B. Timofeyev, A.V. Larionov, J. Hvam, K. Soerensen, Pis'ma v ZhETF, 76, 526 (2002) [JETP Letters 76, 450 (2002)].

5. E.g., see J.P. Eisenstein in Perspectives in Quantum Hall Effects edited by S. Das Sarma and A. Pinczuk (Wiley, New York, 1997).

6. A.H. MacDonald and E.H. Rezayi, Phys. Rev. B 42, 3224 (1990).

7. I.B. Spielman, J.P. Eisenstein, L.N. Pfeiffer, and K.W. West, Phys. Rev. Lett. 84, 5808 (2000).

8. I.B. Spielman, J.P. Eisenstein, L.N. Pfeiffer, and K.W. West, Phys. Rev. Lett. 87, 036803 (2001).

9. M.M. Fogler and F. Wilczek, Phys. Rev. Lett. 86, 1833 (2001).

10. L. Balents and L. Radzikhovsky, Phys. Rev. Lett. 86, 1825 (2001).

11. A. Stern, S.M. Girvin, A.H. MacDonald, and N. Ma, Phys. Rev. Lett. 86, 1829 (2001).

12. G.N. Joglekar and A.H. MacDonald, Phys. Rev. Lett. 87, 196802 (2001).

13. M. Abolfath, R. Khomeriki, and K. Mullen, cond-mat/0208236.

14. R.R. Gusejnov and L.V. Keldysh have first demonstrated that the interband transitions lift the phase degeneracy of the wave function of the electron- hole condensate in the exciton dielectric: R.R. Gusejnov and L.V. Keldysh, Zh. Eksp. Teor. Fiz. 63, 2255 (1972) [Sov. Phys. JETP 36, 1193 (1972)].

15. I.O. Kulik and S.I. Shevchenko, Fiz. Nizk. Temp. 2, 1406 (1976) [Sov. J. Low Temp. Phys. 2,687 (1976)].

16. S.I. Shevchenko, "Theory of low dimensional superfluidity in Bose- and Fermi-systems", thesis, Kharkov, 1992 (unpublished).

17. Similarly to magnetic impurities in superconductors, the usual (nonmagnetic) impurities and crystal lattice distortions in the n-p system suppress the order parameter and lead to the transition of the system to the gapless state in the narrow range of defect concentrations in the vicinity of the critical concentration. E.g., see A.I. Bezuglyj and S.I. Shevchenko, Fiz. Nizk. Temp. 3, 428 (1977) [Sov. J. Low Temp. Phys. 3, 204 (1977)].

18. A.I. Bezuglyj and S.I. Shevchenko, Fiz. Nizk. Temp. 4, 454 (1978) [Sov. J Low Temp. Phys. 4, 222 (1978)].

19. L.D. Landau, E.M. Lifshitz, Physical Kinetics, Course in Theoretical Physics Vol. 10 (Nauka, Moscow, 1979).

20. L.P. Gor'kov, G.M. Eliashberg, Zh. Eksp. Teor. Fiz. 54, 612 (1968).

21. V.N. Belykh, N.F. Pedersen, and O.H. Soerensen, Phys. Rev. B16, 4853 (1977).

* Electronic address: bezugly@ic.kharkov.ua

† Electronic address: shevchenko@ilt.kharkov.ua 